\title{
Efficient Synthesis of Bibenzyl Derivatives Bearing Pyranyl Moieties: First Total Synthesis of Bauhinol D
}

\author{
Byung Ho Park, Yong Rok Lee, and Sung Hong Kim ${ }^{\dagger}$ \\ School of Chemical Engineering and Technology, Yeungnam University, Gyeongsan 712-749, Korea \\ *E-mail:yrlee@yu.ac.kr \\ ${ }^{\dagger}$ Analysis Research Division, Daegu Center, Korea Basic Science Institute, Daegu 702-701, Korea \\ Received November 5, 2010, Accepted December 7, 2010
}

\begin{abstract}
An efficient and general synthesis of bibenzyl derivatives bearing pyranyl rings was achieved by ethylenediamine diacetate-catalyzed reactions of 3,5-dihydroxybibenzyl with $\alpha, \beta$-unsaturated aldehydes in moderate yields. These reactions provided naturally occurring compounds $\mathbf{1}$ and $\mathbf{2}$ in single step reaction. As an application of this methodology, biologically interesting natural bauhinol D (8) was synthesized by a convergent sequence from readily available benzaldehyde and benzyl phosphonate.
\end{abstract}

Key Words: Bibenzyls, Pyrans, Bauhinol D

\section{Introduction}

Molecules containing the bibenzyl moiety are widely distributed in nature ${ }^{1}$ and possess a variety of biological properties, including antioxidant, ${ }^{2}$ antimicrobial, ${ }^{3}$ anti-HIV-1, ${ }^{4}$ antifungal, ${ }^{5}$ anti-proliferative, ${ }^{6}$ antitumor, ${ }^{7}$ cytotoxic, ${ }^{8}$ neuroprotective, ${ }^{9}$ and antiplatelet aggregation activities. ${ }^{10}$ Many of them also find use as versatile intermediates in organic and natural product syntheses. ${ }^{11}$ Among these, bibenzyl derivatives bearing pyranyl rings, such as compounds $\mathbf{1}$ and $\mathbf{2}$, have been isolated from Radula laxiramea, ${ }^{12}$ while $\mathbf{3}$ and $\mathbf{4}$ were isolated from Lethocolea glossophylla (Fig. 1). ${ }^{13}$ Recently, o-cannabichromene (5) and $o$-cannabicyclol (6) were isolated from Radula appressa and Thysananthus spathulistipus. ${ }^{14}$ These compounds inhibit nitric oxide (NO) production in lipopolysaccharide (LPS)-stimulated RAW 264.7 cells. ${ }^{14}$ Bauhinols A (7) and D (8) were isolated from Bauhinia saccocalyx. ${ }^{15}$ The crude extract of this plant has shown to possess antimalarial and antimycobacterial activities. ${ }^{15}$ Bauhinol A(7) itself exhibits significant cytotoxicity towards NCI-H187, BC, and KB cell lines with $\mathrm{IC}_{50}$ values of $2.7-4.5 \mu \mathrm{g} / \mathrm{mL}$. ${ }^{15}$ These biological activities and properties have

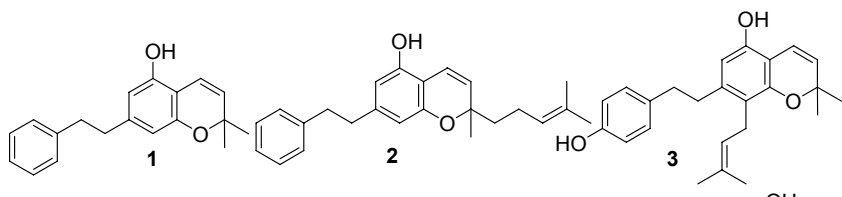<smiles>CC(C)=CCc1c(CCc2ccc(O)cc2)cc2c(c1O)C=CC(C)(C)O2</smiles>
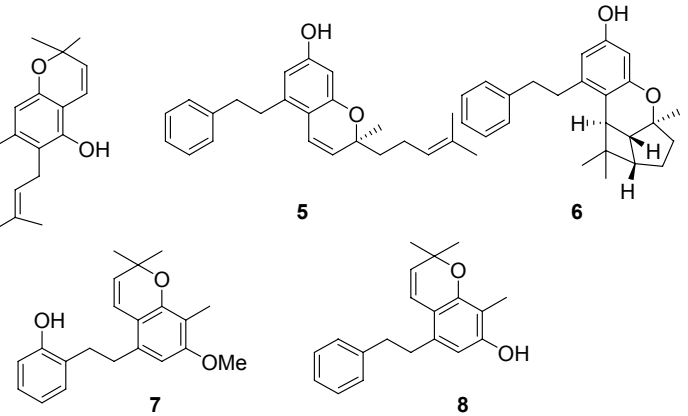

Figure 1. Naturally occurring bibenzyls 1-8 bearing pyranyl rings. stimulated research into the synthesis of bibenzyl derivatives bearing pyranyl rings. The structures of natural products 1-8 have been established by spectral analysis, but no synthesis of naturally occurring bibenzyls 3-8 have been reported yet.

Recently, this lab developed a new and useful methodology for preparing a variety of benzopyrans using ethylenediamine diacetate (EDDA)-catalyzed reactions of resorcinols with $\alpha, \beta$ unsaturated aldehydes through a domino aldol-type/6 $\pi$-electrocyclization reaction. ${ }^{16}$ As part of an ongoing study of the synthetic efficacy of this methodology, we describe herein an efficient and general synthesis of a variety of natural and unnatural bibenzyl derivatives fused with pyranyl rings. Also we report the first total synthesis of the naturally occurring bauhinol D (8).

\section{Results and Discussion}

Initial attempts for the synthesis of 1 employed readily available 3,5-dihydroxybibenzyl (9) (= dihydropinosylvin) as the starting material (Scheme 1). Reaction of 9 with 3-methyl-2butenal in the presence of ethylenediamine diacetate $(20 \mathrm{~mol} \%)$ as a catalyst in refluxing $p$-xylene for $10 \mathrm{~h}$ afforded 1 in $61 \%$ yield. In the ${ }^{1} \mathrm{H}-\mathrm{NMR}$ spectrum, two vinyl protons on the pyranyl ring were observed at $\delta 6.65(1 \mathrm{H}, \mathrm{d}, J=10.2 \mathrm{~Hz})$ and 5.59 $(1 \mathrm{H}, \mathrm{d}, J=10.2 \mathrm{~Hz}) \mathrm{ppm}$. The spectral data of synthetic material 1 were in agreement with those reported in the literature. ${ }^{12}$

Additional reactions of 3,5-dihydroxybibenzyl (9) with several types of $\alpha, \beta$-unsaturated aldehydes such as crotonaldehyde, citral, 1-cyclohexene-1-carboxaldehyde, (-)-perillaldehyde, and (-)-myrtenal were carried out in the presence of EDDA (20 mol \%) in refluxing $p$-xylene (Table 1). Reaction of 9 with crotonaldehyde for $12 \mathrm{~h}$ afforded adduct $\mathbf{1 0}$ in $43 \%$
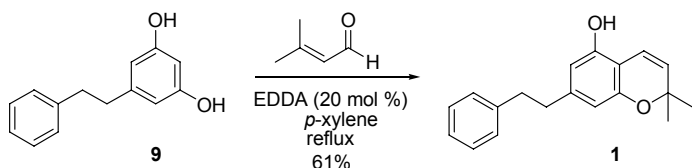

Scheme 1. Reaction of 3,5-dihydroxybibenzyl (9) with 3-methyl-2butenal in the presence of EDDA 
Table 1. Reactions of 3,5-dihydroxybibenzyl (9) with $\alpha, \beta$-unsaturated aldehydes

entry starting material $\alpha, \beta$-unsaturated aldehyde condition

yield, whereas treatment of 9 with citral for $10 \mathrm{~h}$ afforded, interestingly, both naturally occurring compound 2 (32\%) and tetracycle 11 (48\%). The two compounds were easily separated by column chromatography. The spectroscopic data of $\mathbf{2}$ were in agreement with those of the natural product. ${ }^{12}$ Adduct 11 was confirmed by comparison of its spectral data with those of the known authentic compound. ${ }^{17}$ Reactions of 9 with 1-cyclohexene-1-carboxaldehyde and (-)-perillaldehyde in refluxing $p$-xylene provided adducts 12 and $\mathbf{1 3}$ in 60 and $68 \%$ yields, respectively, as a single compound, whereas treatment with

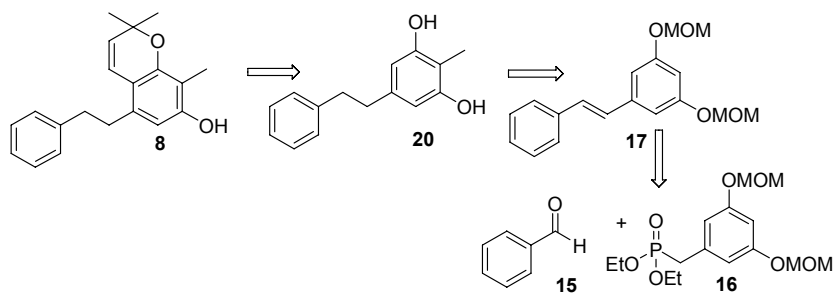

Scheme 2. Retrosynthetic analysis for the synthesis of bauhinol D (8) (-)-myrtenal gave product 14 with $90 \%$ diastereoselectivity in $65 \%$ yield. The stereochemistry of new compounds 13 and 14 was confirmed by comparison with reported data. ${ }^{18}$ These reactions provide a rapid route for the syntheses of bibenzyl derivatives with a variety of substituents on the pyranyl rings.

As an application of this methodology, the total synthesis of bauhinol D (8) was next attempted. Scheme 2 shows the retrosynthetic strategy for naturally occurring 8 . Bauhinol D (8) could be prepared from $\mathbf{2 0}$ by a benzopyran formation reaction. Compound 20 could be generated from 17 by consecutive hydrogenation, alkylation, and deprotection reactions. Compound 17 could be produced via a Horner-Wadsworth-Emmons reaction between benzaldehyde (15) and benzyl phosphonate $\mathbf{1 6}$.

The synthetic approach for bauhinol D (8) is shown in Scheme 3. The Horner-Wadsworth-Emmons reaction of benzaldehyde 15 with benzyl phosphonate $16^{19}$ provided trans-stilbene $\mathbf{1 7}$ in $85 \%$ yield. Catalytic hydrogenation of 17 over $\mathrm{Pd} / \mathrm{C}$ (30 psi) in ethyl acetate for $1 \mathrm{~h}$ gave $\mathbf{1 8}$ in $96 \%$ yield. Reaction of 18 with $n$-BuLi, followed by the addition of methyl iodide, provided compound 19 in $95 \%$ yield. Deprotection of the two

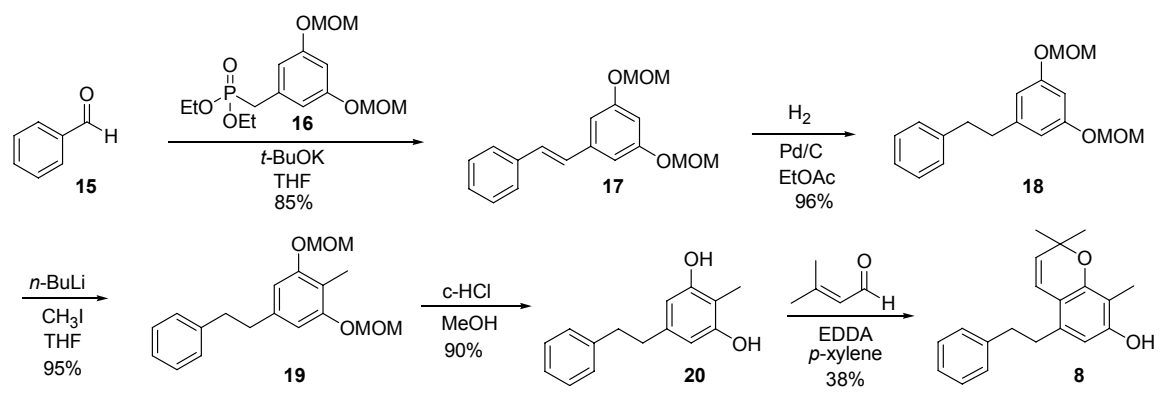

Scheme 3 
MOM ethers with concentrated $\mathrm{HCl}$ in methanol afforded compound $20(90 \%)$, known as stilbostemin B. This compound was isolated from Stemona collinsae $e^{20}$ and S. sessilifolia, ${ }^{21}$ which have long been used in traditional Chinese and Vietnamese medicines for the treatment of inflammatory related diseases. "Baibu", the dried root tuber of Stemona sessilifolia, is listed in the Chinese Pharmacopoeia and used to relieve cough and kill insects and worms. ${ }^{22}$ In Vietnamese medicine, S. collinsae has been used for cough relief and as an antiasthmatic. ${ }^{23}$ Naturally occurring stilbostemin B (20) showed strong antifungal ${ }^{24}$ and antibacterial $^{25}$ activities, along with inhibition of leukotriene formation. ${ }^{27}$ Treatment of $\mathbf{2 0}$ with 3-methyl-2-butenal in the presence of $20 \mathrm{~mol} \%$ EDDA as a catalyst in refluxing xylene for $12 \mathrm{~h}$ gave adduct 8 (38\%). The spectroscopic data of synthetic $\mathbf{8}$ were in agreement with those of the literature. ${ }^{15}$

In conclusion, the formation of a number of natural and unnatural bibenzyl derivatives with a fused pyran moiety has been described starting from 3,5-dihydroxybibenzyl with $\alpha, \beta$-unsaturated aldehydes in the presence of ethylenediamine diacetate. As an application of this methodology, the total synthesis of naturally occurring bauhinol D (8) was achieved from benzaldehyde and benzyl phosphonate through Horner-WadsworthEmmons olefination and benzopyran formation reaction as key steps.

\section{Experimental Section}

All experiments were carried out in a nitrogen atmosphere. Merck, pre-coated silica gel plates (Art. 5554) with a fluorescent indicator were used for TLC analysis. Flash column chromatography was performed using silica gel 9385 (Merck). The ${ }^{1} \mathrm{H}$ and ${ }^{13} \mathrm{C}-\mathrm{NMR}$ spectra were recorded using a Bruker Model $\mathrm{ARX}$ (300 and $75 \mathrm{MHz}$, respectively) spectrometer in $\mathrm{CDCl}_{3}$ as the solvent for the chemical shift. The IR spectra were recorded using a Jasco FTIR 5300 spectrophotometer. HRMS spectra were carried out at the Korea Basic Science Institute.

General Procedure for the Synthesis of 1, 2, and 10-14. 3,5Dihydroxybibenzyl $(0.5 \mathrm{mmol})$ and $\alpha, \beta$-unsaturated aldehydes $(1 \mathrm{mmol})$ were dissolved in $p$-xylene $(10 \mathrm{~mL})$, and ethylenediamine diacetate $(18 \mathrm{mg}, 0.1 \mathrm{mmol})$ was added at room temperature. The mixture was heated to reflux for $10-12 \mathrm{~h}$ and then cooled to room temperature. Removal of the solvent at reduced pressure left an oily residue, which was then purified by column chromatography on silica gel to give the product.

2,2-Dimethyl-7-phenethyl-2H-chromen-5-ol (1): Reaction of 9 (107 mg, $0.5 \mathrm{mmol}$ ) with 3-methyl-2-butenal (84 mg, 1.0 $\mathrm{mmol})$ in xylene $(10 \mathrm{~mL})$ afforded $\mathbf{1}(86 \mathrm{mg}, 61 \%)$ as an oil. ${ }^{1} \mathrm{H}$ NMR $\left(300 \mathrm{MHz}, \mathrm{CDCl}_{3}\right) \delta$ 7.35-7.33 (2H, m), 7.26-.21 $(3 \mathrm{H}, \mathrm{m}), 6.65(1 \mathrm{H}, \mathrm{d}, J=10.2 \mathrm{~Hz}), 6.35(1 \mathrm{H}, \mathrm{s}), 6.21(1 \mathrm{H}, \mathrm{s})$, $5.59(1 \mathrm{H}, \mathrm{d}, J=10.2), 2.95-2.87(2 \mathrm{H}, \mathrm{m}), 2.85-2.78(2 \mathrm{H}, \mathrm{m})$, $1.49(6 \mathrm{H}, \mathrm{s}) ;{ }^{13} \mathrm{C} \mathrm{NMR}\left(75 \mathrm{MHz}, \mathrm{CDCl}_{3}\right) \delta 153.8,151.2,143.5$, 141.7, 128.4, 128.3, 125.9, 116.4, 109.1, 107.9, 107.4, 75.9, $37.9,37.4,27.8$; IR (neat) 3462, 2974, 1622, 1570, 1429, 1424, $1180,1122,1064,746,700 \mathrm{~cm}^{-1}$; HRMS $m / z\left(\mathrm{M}^{+}\right)$calcd for $\mathrm{C}_{19} \mathrm{H}_{20} \mathrm{O}_{2}: 280.1463$. Found: 280.1466 .

2-Methyl-7-phenethyl-2H-chromen-5-ol (10): Reaction of 9 (107 mg, $0.5 \mathrm{mmol})$ with crotonaldehyde (70 mg, $1 \mathrm{mmol})$ in xylene $(10 \mathrm{~mL})$ afforded $10(57 \mathrm{mg}, 43 \%)$ as an oil. ${ }^{1} \mathrm{H}$ NMR $\left(300 \mathrm{MHz}, \mathrm{CDCl}_{3}\right) \delta 7.29-7.15(5 \mathrm{H}, \mathrm{m}), 6.63(1 \mathrm{H}, \mathrm{d}, J=10.2$ $\mathrm{Hz}), 6.29(1 \mathrm{H}, \mathrm{s}), 6.11(1 \mathrm{H}, \mathrm{s}), 5.58(1 \mathrm{H}, \mathrm{d}, J=10.2 \mathrm{~Hz}), 4.90-$ $4.80(1 \mathrm{H}, \mathrm{m}), 2.88-2.72(4 \mathrm{H}, \mathrm{m}), 1.42(3 \mathrm{H}, \mathrm{d}, J=6.6 \mathrm{~Hz}) ;{ }^{13} \mathrm{C}$ NMR $\left(75 \mathrm{MHz}, \mathrm{CDCl}_{3}\right) \delta 154.0,151.0,143.6,141.8,128.4$, 128.3, 125.9, 124.5, 118.0, 108.8, 108.2, 71.2, 37.8, 37.3, 21.0; IR (KBr) 3431, 2931, 1617, 1594, 1492, 1438, 1366, 1206, $1141,1065,918,846,737 \mathrm{~cm}^{-1}$; HRMS $m / z\left(\mathrm{M}^{+}\right)$calcd for $\mathrm{C}_{18} \mathrm{H}_{18} \mathrm{O}_{2}$ : 266.1307. Found: 266.1306.

2-Methyl-2-(4-methyl-pent-3-enyl)-7-phenethyl-2H-chromen-5-ol (2) and 2-Methyl-2-(4-methyl-pent-3-enyl)-7-phenethyl-2H-chromen-5-ol (11): Reaction of 9 (107 mg, $0.5 \mathrm{mmol})$ with citral (152 mg, $1 \mathrm{mmol})$ in xylene $(10 \mathrm{~mL})$ afforded $2(56$ $\mathrm{mg}, 32 \%)$ and 11 (84 mg, 48\%). Compound 2: ${ }^{1} \mathrm{H}$ NMR (300 $\left.\mathrm{MHz}, \mathrm{CDCl}_{3}\right) \delta$ 7.29-7.24 (2H, m), 7.20-7.15 (3H, m), $6.62(1 \mathrm{H}$, $\mathrm{d}, J=10.2 \mathrm{~Hz}), 6.27(1 \mathrm{H}, \mathrm{s}), 6.11(1 \mathrm{H}, \mathrm{s}), 5.49(1 \mathrm{H}, \mathrm{d}, J=10.2$ $\mathrm{Hz}), 5.09(1 \mathrm{H}, \mathrm{t}, J=7.0 \mathrm{~Hz}), 4.97(1 \mathrm{H}, \mathrm{br} \mathrm{s}), 2.88-2.82(2 \mathrm{H}$, $\mathrm{m}), 2.77-2.71(2 \mathrm{H}, \mathrm{m}), 2.14-2.04(2 \mathrm{H}, \mathrm{m}), 1.77-1.67(2 \mathrm{H}, \mathrm{m})$, $1.65(3 \mathrm{H}, \mathrm{s}), 1.57(3 \mathrm{H}, \mathrm{s}), 1.37(3 \mathrm{H}, \mathrm{s}) ;{ }^{13} \mathrm{C} \mathrm{NMR}(75 \mathrm{MHz}$, $\left.\mathrm{CDCl}_{3}\right) \delta 154.1,151.2,143.5,141.8,131.7128 .3,128.3,127.3$, 125.9, 124.1, 116.8, 109.0, 107.7, 107.3, 78.2, 41.0, 38.1, 37.3, 26.1, 25.7, 22.7, 17.6; IR (neat) 3396, 3028, 2926, 1624, 1577, 1496, 1433, 1375, 1253, 1203, 1141, 1084, 1057, 904, 823, $775,700 \mathrm{~cm}^{-1}$; HRMS $m / z\left(\mathrm{M}^{+}\right)$calcd for $\mathrm{C}_{24} \mathrm{H}_{28} \mathrm{O}_{2}: 348.2089$. Found: 348.2089. Compound 11: ${ }^{1} \mathrm{H} \mathrm{NMR}\left(300 \mathrm{MHz}, \mathrm{CDCl}_{3}\right)$ 反 7.26-7.21 (2H, m), 7.16-7.13 (3H, m), $6.34(1 \mathrm{H}, \mathrm{s}), 6.25(1 \mathrm{H}$, s), 2.96-2.60 $(4 \mathrm{H}, \mathrm{m}), 2.24-2.17(1 \mathrm{H}, \mathrm{m}), 2.04-1.97(1 \mathrm{H}, \mathrm{m})$, $1.82(1 \mathrm{H}, \mathrm{d}, J=13.2 \mathrm{~Hz}), 1.50(3 \mathrm{H}, \mathrm{s}), 1.46-1.39(2 \mathrm{H}, \mathrm{m}), 1.36$ $(3 \mathrm{H}, \mathrm{s}), 1.25-1.18(2 \mathrm{H}, \mathrm{m}), 0.98(3 \mathrm{H}, \mathrm{s}), 0.63-0.58(1 \mathrm{H}, \mathrm{m})$; ${ }^{13} \mathrm{C} \mathrm{NMR}\left(75 \mathrm{MHz}, \mathrm{CDCl}_{3}\right) \delta 157.0,156.7,141.9,141.3,128.4$, 128.2, 125.8, 114.4, 109.8, 108.9, 83.6, 74.5, 46.8, 38.1, 37.8, $37.3,35.3,29.7,29.0,28.1,23.8,22.1$; IR (neat) 2974, 1620, $1585,1431,1367,1211,1163,1128,1062,700 \mathrm{~cm}^{-1}$; HRMS $m / z\left(\mathrm{M}^{+}\right)$calcd for $\mathrm{C}_{24} \mathrm{H}_{28} \mathrm{O}_{2}: 348.2089$. Found: 348.2087.

3-Phenethyl-5,7,8,10a-tetrahydro-6H-xanthen-1-ol (12): Reaction of 9 (107 mg, $0.5 \mathrm{mmol})$ with 1-cyclohexene-1-carboxaldehyde (110 mg, $1 \mathrm{mmol})$ in xylene $(10 \mathrm{~mL})$ afforded 12 (92 mg, 60\%) as an oil. ${ }^{1} \mathrm{H} \mathrm{NMR}\left(300 \mathrm{MHz}, \mathrm{CDCl}_{3}\right) \delta$ 7.29-7.24 $(2 \mathrm{H}, \mathrm{m}), 7.20-7.15(3 \mathrm{H}, \mathrm{m}), 6.28(1 \mathrm{H}, \mathrm{s}), 6.21(1 \mathrm{H}, \mathrm{s}), 6.07$ $(1 \mathrm{H}, \mathrm{s}), 4.91-4.85(1 \mathrm{H}, \mathrm{m}), 4.68(1 \mathrm{H}, \mathrm{br} \mathrm{s}), 2.87-2.80(2 \mathrm{H}, \mathrm{m})$, 2.75-2.69 (2H, m), 2.48-2.41 (1H, m), 2.24-2.00 (2H, m), 1.91$1.66(3 \mathrm{H}, \mathrm{m}), 1.52-1.25(2 \mathrm{H}, \mathrm{m}) ;{ }^{13} \mathrm{C} \mathrm{NMR}\left(75 \mathrm{MHz}, \mathrm{CDCl}_{3}\right)$ $\delta 153.8,150.2,142.2,141.7,135.6,128.4,128.3,125.9,110.0$, 108.0, 107.9, 107.2, 77.4, 37.8, 37.4, 35.0, 33.1, 26.7, 24.3; IR (neat) 3402, 3026, 2930, 2856, 1628, 1583, 1496, 1434, 1346, $1161,1034,931,847,739 \mathrm{~cm}^{-1}$; HRMS $m / z\left(\mathrm{M}^{+}\right)$calcd for $\mathrm{C}_{21} \mathrm{H}_{22} \mathrm{O}_{2}: 306.1620$. Found: 306.1616 .

6-Isopropenyl-3-phenethyl-5,7,8,10a-tetrahydro-6H-xanthen-1-ol (13): Reaction of 9 (107 mg, $0.5 \mathrm{mmol})$ with (-)perillaldehyde $(150 \mathrm{mg}, 1 \mathrm{mmol})$ in xylene $(10 \mathrm{~mL})$ afforded $13(118 \mathrm{mg}, 68 \%)$ as an oil. ${ }^{1} \mathrm{H}$ NMR $\left(300 \mathrm{MHz}, \mathrm{CDCl}_{3}\right) \delta$ 7.28-7.24 (2H, m), 7.19-7.15 (3H, m), $6.32(1 \mathrm{H}, \mathrm{s}), 6.22(1 \mathrm{H}$, s), $6.07(1 \mathrm{H}, \mathrm{s}), 4.99-4.91(1 \mathrm{H}, \mathrm{m}), 4.79(1 \mathrm{H}$, br s $), 4.73(2 \mathrm{H}$, s), 2.87-2.82 (2H, m), 2.74-2.69 $(2 \mathrm{H}, \mathrm{m}), 2.53-2.48(1 \mathrm{H}, \mathrm{m})$, 2.28-2.23 (1H, m), 2.19-2.10 (2H, m), 1.86-1.76 (2H, m), 1.74 $(3 \mathrm{H}, \mathrm{s}), 1.40-1.24(1 \mathrm{H}, \mathrm{m}) ;{ }^{13} \mathrm{C} \mathrm{NMR}\left(75 \mathrm{MHz}, \mathrm{CDCl}_{3}\right) \delta 153.8$, $150.3,148.4,142.4,141.7,135.2,134.6,128.4,125.9,110.3$, 109.3, 108.1, 107.9, 107.4, 76.6, 43.2, 39.7, 37.7, 37.4, 32.4, $31.7,20.7$; IR (neat) 3376, 3063, 2934, 2346, 1612, 1580, 1498, 
$1432,1352,1277,1165,1045,963,890,738 ;$ HRMS $m / z\left(\mathrm{M}^{+}\right)$ calcd for $\mathrm{C}_{24} \mathrm{H}_{26} \mathrm{O}_{2}$ : 346.1933. Found: 346.1933.

14,14-Dimethyl-7-(2-phenylethyl)-10-oxatetracyclo[11.1. $1.0^{2,11} .0^{4,9}$ ]pentadeca-2,4(9),5,7-tetraen-5-ol (14): Reaction of 9 (107 mg, $0.5 \mathrm{mmol}$ ) with (-)-myrtenal (150 mg, $1 \mathrm{mmol})$ in xylene $(10 \mathrm{~mL})$ afforded $14(113 \mathrm{mg}, 65 \%)$ as a 95:5 mixture of diastereomers. Major: ${ }^{1} \mathrm{H} \mathrm{NMR}\left(300 \mathrm{MHz}, \mathrm{CDCl}_{3}\right) \delta 7.29$ $7.24(2 \mathrm{H}, \mathrm{m}), 7.19-7.16(3 \mathrm{H}, \mathrm{m}), 6.39(1 \mathrm{H}, \mathrm{s}), 6.27(1 \mathrm{H}, \mathrm{s})$, $6.19(1 \mathrm{H}, \mathrm{s}), 4.98-4.92(1 \mathrm{H}, \mathrm{m}), 2.88-2.83(2 \mathrm{H}, \mathrm{m}), 2.80-2.75$ $(2 \mathrm{H}, \mathrm{m}), 2.64-2.52(2 \mathrm{H}, \mathrm{m}), 2.41-2.33(1 \mathrm{H}, \mathrm{m}), 2.15-2.02(4 \mathrm{H}$, m), $1.29(3 \mathrm{H}, \mathrm{s}), 0.90(3 \mathrm{H}, \mathrm{s}) ;{ }^{13} \mathrm{C} \mathrm{NMR}\left(75 \mathrm{MHz}\right.$, acetone- $\left.d_{6}\right)$ $\delta 155.4,150.8,141.9,141.7,139.6,128.4,128.3,125.9,111.1$, 110.0, 108.7, 108.5, 69.9, 49.1, 42.1, 40.3, 37.8, 37.5, 31.9, 25.9, 25.6, 21.8; IR (KBr) 3372, 2932, 1700, 1576, 1502, 1434, 1353, 1206, 1049, 962, 912, 829, 737, $697 \mathrm{~cm}^{-1}$; HRMS m/z $\left(\mathrm{M}^{+}\right)$ calcd for $\mathrm{C}_{24} \mathrm{H}_{26} \mathrm{O}_{2}$ : 346.1933. Found: 346.1933. Minor: ${ }^{1} \mathrm{H}$ NMR $\left(300 \mathrm{MHz}, \mathrm{CDCl}_{3}\right) \delta$ 7.29-7.24 (2H, m), 7.19-7.16 (3H, $\mathrm{m}), 6.42(1 \mathrm{H}, \mathrm{s}), 6.27(1 \mathrm{H}, \mathrm{s}), 6.22(1 \mathrm{H}, \mathrm{s}), 4.83-4.78(1 \mathrm{H}, \mathrm{m})$, 2.88-2.83 (2H, m), 2.80-2.75 (2H, m), 2.64-2.52 (2H, m), 2.41$2.33(1 \mathrm{H}, \mathrm{m}), 2.15-2.02(4 \mathrm{H}, \mathrm{m}), 1.30(3 \mathrm{H}, \mathrm{s}), 0.83(3 \mathrm{H}, \mathrm{s})$; ${ }^{13} \mathrm{C}$ NMR $\left(75 \mathrm{MHz}\right.$, acetone- $\left.d_{6}\right) \delta 156.0,151.4,142.2,140.6$, $140.0,128.4,128.3,125.9,111.4,110.7,108.7,108.3,70.6$, 50.5, 41.6, 39.8, 37.8, 37.5, 35.3, 33.4, 26.3, 23.7 .

(E)-3,5-Bis(methoxymethoxy)stilbene (17): To a solution of $15(0.318 \mathrm{~g}, 3.0 \mathrm{mmol})$ and benzyl phosphonate 16 (1.045 g, $3.0 \mathrm{mmol})$ in THF $(30 \mathrm{~mL})$ was added $t$-BuOK $(0.717 \mathrm{~g}, 6.4$ mmol) at $0{ }^{\circ} \mathrm{C}$. The reaction mixture was stirred at $0{ }^{\circ} \mathrm{C}$ for 30 $\mathrm{min}$. The reaction mixture was quenched by addition of water $(30 \mathrm{~mL})$ and extracted with EtOAc $(3 \times 30 \mathrm{~mL})$. The combined organic layer was washed with $\mathrm{NH}_{4} \mathrm{Cl}$ solution $(30 \mathrm{~mL})$, water $(30 \mathrm{~mL})$, brine $(30 \mathrm{~mL})$, dried over $\mathrm{MgSO}_{4}$, and concentrated at reduced pressure. The removal of the solvent under reduced pressure left an oily residue, which was then purified by column chromatography on silica gel using hexane/ethyl acetate (20:1) to give product $17(0.766 \mathrm{~g}, 85 \%)$ as an oil: ${ }^{1} \mathrm{H} \mathrm{NMR}(300 \mathrm{MHz}$, $\left.\mathrm{CDCl}_{3}\right) \delta 7.41(2 \mathrm{H}, \mathrm{d}, J=7.2 \mathrm{~Hz}), 7.26(2 \mathrm{H}, \mathrm{dd}, J=7.5,7.2$ $\mathrm{Hz}), 7.17(1 \mathrm{H}, \mathrm{t}, J=7.5 \mathrm{~Hz}), 7.01(1 \mathrm{H}, \mathrm{d}, J=16.0 \mathrm{~Hz}), 6.93$ $(1 \mathrm{H}, \mathrm{d}, J=16.0 \mathrm{~Hz}), 6.79(2 \mathrm{H}, \mathrm{s}), 6.57(1 \mathrm{H}, \mathrm{s}), 5.01(4 \mathrm{H}, \mathrm{s})$, $3.41(6 \mathrm{H}, \mathrm{s}) ;{ }^{13} \mathrm{C} \mathrm{NMR}\left(75 \mathrm{MHz}, \mathrm{CDCl}_{3}\right) \delta 158.5,139.5,137.0$, 129.3, 128.6, 128.3 127.7, 126.5, 107.8, 104.2, 94.4, 56.0; IR (neat) 2953, 2825, 1591, 1454, 1400, 1282, 1213, 1147, 1084, $1035,962,923,841,752 \mathrm{~cm}^{-1}$; HRMS $\mathrm{m} / z\left(\mathrm{M}^{+}\right)$calcd for $\mathrm{C}_{18} \mathrm{H}_{20} \mathrm{O}_{4}: 300.1362$. Found: 300.1364 .

1,3-Bis(methoxymethoxy)-5-styrylbenzene (18): To a solution of $17(0.451 \mathrm{~g}, 1.5 \mathrm{mmol})$ in ethyl acetate $(10 \mathrm{~mL})$ was added $\mathrm{Pd} / \mathrm{C}(10 \mathrm{wt} \%, 0.05 \mathrm{~g})$ and the suspension was hydrogenated over $30 \mathrm{psi}$ for $1 \mathrm{~h}$ at room temperature. The reaction mixture was filtered through celite and removal of the solvent at reduced pressure left the residue, which was then purified by column chromatography on silica gel with hexane/EtOAc (20:1) to give $18(0.435 \mathrm{~g}, 96 \%)$ as an oil. ${ }^{1} \mathrm{H} \mathrm{NMR}(300 \mathrm{MHz}$, $\left.\mathrm{CDCl}_{3}\right) \delta$ 7.28-7.24 (2H, m), 7.20-7.05 (3H, m), $6.58(1 \mathrm{H}, \mathrm{d}, J=$ $2.1 \mathrm{~Hz}), 6.60(2 \mathrm{H}, \mathrm{d}, J=2.1 \mathrm{~Hz}), 5.18(4 \mathrm{H}, \mathrm{s}), 3.48(6 \mathrm{H}, \mathrm{s})$, $2.85\left(4 \mathrm{H}\right.$, br s) ${ }^{13} \mathrm{C}$ NMR $\left(75 \mathrm{MHz}, \mathrm{CDCl}_{3}\right) \delta 158.1,144.3$, 141.6, 128.3, 125.8, 109.8, 102.4, 94.3, 56.0, 38.1, 37.6; IR (neat) 2951, 1597, 1456, 1400, 1282, 1215, 1147, 1084, 1037, 923, $848 \mathrm{~cm}^{-1}$; HRMS m/z $\left(\mathrm{M}^{+}\right)$calcd for $\mathrm{C}_{18} \mathrm{H}_{22} \mathrm{O}_{4}: 302.1518$. Found: 302.1520 .
1,3-Bis(methoxymethoxy)-2-methyl-5-styrylbenzene (19): $n$-BuLi $(0.44 \mathrm{~mL}, 2.5 \mathrm{M}$ in hexane, $1.1 \mathrm{mmol})$ was added at $0{ }^{\circ} \mathrm{C}$ to a solution of $\mathbf{1 8}(0.302 \mathrm{~g}, 1.0 \mathrm{mmol})$ in THF $(20 \mathrm{~mL})$ and the resulting solution was stirred at $0{ }^{\circ} \mathrm{C}$ for $2 \mathrm{~h}$. Methyl iodide $(0.156 \mathrm{~g}, 1.1 \mathrm{mmol})$ was added dropwise to the reaction mixture at $0{ }^{\circ} \mathrm{C}$, which was stirred at room temperature for $10 \mathrm{~h}$. The reaction mixture was quenched with saturated $\mathrm{NH}_{4} \mathrm{Cl}$ solution $(20 \mathrm{~mL})$ and extracted with ethyl acetate $(3 \times 30 \mathrm{~mL})$. The combined extracts were washed water $(30 \mathrm{~mL})$, dried $\left(\mathrm{MgSO}_{4}\right)$, and evaporated under reduced pressure. Flash chromatography on silica gel using hexane/ethyl acetate (10:1) afforded 19 (0.301 g, 95\%) as an oil. ${ }^{1} \mathrm{H} \mathrm{NMR}\left(300 \mathrm{MHz}, \mathrm{CDCl}_{3}\right)$ 7.28-7.24 $(2 \mathrm{H}, \mathrm{m}), 7.19-7.17(3 \mathrm{H}, \mathrm{m}), 6.58(2 \mathrm{H}, \mathrm{s}), 5.14(4 \mathrm{H}, \mathrm{s}), 3.47(6 \mathrm{H}$, s), $2.87(4 \mathrm{H}$, br s $), 2.12(3 \mathrm{H}, \mathrm{s}) ;{ }^{13} \mathrm{C} \mathrm{NMR}\left(75 \mathrm{MHz}, \mathrm{CDCl}_{3}\right) \delta$ $155.9,141.7,140.4,128.5,128.2,125.8,114.2$, 108.3, 94.7, 56.0, 38.1, 37.9, 8.5; IR (neat) 2931, 1593, 1446, 1296, 1208, $1111,1060,997,924,837,741 \mathrm{~cm}^{-1}$; HRMS $m / z\left(\mathrm{M}^{+}\right)$calcd for $\mathrm{C}_{19} \mathrm{H}_{24} \mathrm{O}_{4}$ : 316.1675 . Found: 316.1675 .

Stibostemin B (20): To a solution of $19(0.158 \mathrm{~g}, 0.5 \mathrm{mmol})$ in methanol $(5 \mathrm{~mL})$ was added $\mathrm{c}-\mathrm{HCl}(0.2 \mathrm{~mL})$ and the reaction mixture was stirred at room temperature for $10 \mathrm{~h}$. The reaction mixture was diluted with saturated $\mathrm{NaHCO}_{3}$ solution $(30 \mathrm{~mL})$ and extracted with EtOAc $(3 \times 30 \mathrm{~mL})$. Removal of solvent at reduced pressure left an oily residue, which was then purified by column chromatography on silica gel with hexane/EtOAc $(5: 1)$ to give $20(0.103 \mathrm{~g}, 90 \%)$ as a solid. $\mathrm{mp} 153-154{ }^{\circ} \mathrm{C} .{ }^{1} \mathrm{H}$ NMR $\left(300 \mathrm{MHz}, \mathrm{CDCl}_{3}\right) \delta$ 7.29-7.24 $(2 \mathrm{H}, \mathrm{m}), 7.23-7.15(3 \mathrm{H}$, m), $6.23(2 \mathrm{H}, \mathrm{s}), 4.65(1 \mathrm{H}, \mathrm{br} \mathrm{s}), 2.89-2.72(4 \mathrm{H}, \mathrm{m}), 2.10(3 \mathrm{H}$, $\mathrm{s}) ;{ }^{13} \mathrm{C} \mathrm{NMR}\left(75 \mathrm{MHz}, \mathrm{CDCl}_{3}\right) \delta 155.4,141.9,140.0,128.3$, 128.1, 125.6, 108.3, 107.0, 37.5, 37.4, 8.0; (KBr) 3388, 3023, 2926, 1634, 1573, 1500, 1453, 1343, 1289, 1176, 1087, 854, 742; HRMS $m / z\left(\mathrm{M}^{+}\right)$calcd for $\mathrm{C}_{15} \mathrm{H}_{16} \mathrm{O}_{2}: 228.1150$. Found: 228.1149 .

Bauhinol D (8): To a solution of $20(0.068 \mathrm{~g}, 0.3 \mathrm{mmol})$ and 3-methyl-2-butenal $(0.034 \mathrm{~g}, 0.4 \mathrm{mmol})$ in $p$-xylene $(10 \mathrm{~mL})$ was added ethylenediamine diacetate $(11 \mathrm{mg}, 0.06 \mathrm{mmol})$ at room temperature. The reaction mixture was refluxed for $12 \mathrm{~h}$ and then cooled to room temperature. Evaporation of solvent and purification by column chromatography on silica gel using hexane/ethylacetate (4:1) gave $8(0.034 \mathrm{~g}, 38 \%)$. ${ }^{1} \mathrm{H}$ NMR (300 $\left.\mathrm{MHz}, \mathrm{CDCl}_{3}\right) \delta$ 7.29-7.24 (2H, m), 7.20-7.15 (3H, m), $6.45(1 \mathrm{H}$, $\mathrm{d}, J=10.2 \mathrm{~Hz}), 6.18(1 \mathrm{H}, \mathrm{s}), 5.50(1 \mathrm{H}, \mathrm{d}, J=10.2 \mathrm{~Hz}), 4.78$ (1H, br s), $2.80(4 \mathrm{H}, \mathrm{m}), 2.06(3 \mathrm{H}, \mathrm{s}), 1.39(6 \mathrm{H}, \mathrm{s}) ;{ }^{13} \mathrm{C} \mathrm{NMR}$ $\left(75 \mathrm{MHz}, \mathrm{CDCl}_{3}\right) \delta 154.0,152.2,141.7,135.5,128.4,128.3$, 127.6, 126.0, 119.2, 112.6, 109.8, 108.0, 75.3, 37.6, 34.2, 27.7, 7.7; IR (neat) 3406, 2970, 2928, 1598, 1495, 1452, 1420, 1320, $1265,1103,743, \mathrm{~cm}^{-1}$; HRMS $m / z\left(\mathrm{M}^{+}\right)$calcd for $\mathrm{C}_{20} \mathrm{H}_{22} \mathrm{O}_{2}$ : 294.1620. Found: 294.1621.

Acknowledgments. This work was supported by a Yeungnam University research grant, 210-A-380-040.

\section{References}

1. (a) Dong, H.-L.; Wang, C.-L.; Guo, S.-X.; Yang, J.-S. Chem. Pharm. Bull. 2009, 57, 513. (b) Feng, J.-Q.; Zhang, R.-J.; Zhao, W.-M. Helv. Chim. Acta 2008, 91, 520. (c) Lin, Y.-L.; Chen, W.-P. Chem. Pharm. Bull. 2005, 53, 1111. (d) Hashimoto, T.; Irita, H.; Takaoka, S.; Tanaka, M.; Asakawa, Y. Tetrahedron 2000, 56, 3153. 
(e) Oiso, Y.; Toyota, M.; Asakawa, Y. Chem. Pharm. Bull. 1999, 47, 297. (f) Zidorn, C.; Lohwasser, U.; Pschorr, S.; Salvenmoser, D.; Ongania, K.-H.; Ellmerer, E. P.; Börner, A.; Stuppner, H. Phytochemistry 2005, 66, 1691. (g) Fang, L.; Guo, H.-F.; Lou, H.-X. Helv. Chim. Acta 2007, 90, 748. (h) Liu, Q.-F.; Chen, W.-L.; Tang, J.; Zhao, W.-M. Helv. Chim. Acta 2007, 90, 1745. (i) Bardón, A.; Mitre, G. B.; Kamiya, N.; Toyota, M.; Asakawa, Y. Phytochemistry 2002, 59, 205. (j) von Reuß, S. H.; König, W. Phytochemistry 2004, 65, 3113. (k) Fan, C.; Wang W.; Wang, Y.; Qin, G.; Zhao, W. Phytochemistry 2001, 57, 1255 .

2. (a) Zhang, X.; Xu, J.-K.; Wang, J.; Wang, N.-L.; Kurihara, H.; Kitanaka, S.; Yao, X.-S. J. Nat. Prod. 2007, 70, 24. (b) Li, Y.; Wang, C.-L.; Wang, Y.-J.; Wang, F.-F.; Guo, S,-X.; Yang, J.-S.; Xiao, P.-G. Chem. Pharm. Bull. 2009, 57, 997. (c) Yi, Y.; Wang, C.-L.; Wang, Y.-J.; Guo, S.-X.; Yang, J.-S.; Chen, X.-M.; Xiao, P.-G. Chem. Pharm. Bull. 2009, 57, 218.

3. Beck, S.-H.; Phipps, R. K.; Perry, N. J. Nat. Prod. 2004, 67, 718.

4. Manfredi, K. P.; Vallurupalli, V.; Demidova, M.; Kindscher, K.; Pannell, L. K. Phytochemistry 2001, 58, 153.

5. (a) Wentao, Y.; Lou, H. Phytochemistry 2007, 68, 1767. (b) Niu, C.; Qu, J.-B.; Lou, H.-X. Chem. Biodives. 2006, 3, 34. (c) Zhang, Y.-Z.; Xu, G.-B.; Zhang, T. J. Asian, Nat. Prod. Res. 2008, 10, 634.

6. Zhang, W.-G.; Zhao, R.; Ren, J.; Ren, L.-X.; Lin, J.-G.; Liu, D.-L.; Wu, Y.-L.; Yao, X.-S. Arch. Pharm. 2007, 340, 244.

7. Speicher, A.; Groh, M.; Zapp, J.; Schaumloeffel, A.; Knauer, M.; Bringmann, G. Synlett 2009, 1852.

8. Scher, J. S.; Burgess, E. J.; Lorimer, S. D.; Perry, N. B. Tetrahedron 2002, 58,7875 .

9. Lee, K. Y.; Sung, S. H.; Kim, Y. C. J. Nat. Prod. 2006, 69, 679.

10. Hu, J.-M.; Chen, J.-J.; Yu, H.; Zhao, Y.-X.; Zhou, J. J. Asian, Nat. Prod. Res. 2008, 10, 647

11. (a) Song, Y.; Hwang, S.; Gong, P.; Kim, D.; Kim, S. Org. Lett. 2008, 10, 269. (b) Eicher, T.; Tiefensee, K.; Döing, R.; Pick R. Synthesis 1991, 98. (c) Kuzmanich, G.; Gard, M. N.; Garcia-Garibay, M. A. J. Am. Chem. Soc. 2009, 131, 11606. (d) Chesta, C. A.; Mohanty, J.; Nau, W. M.; Bhattacharjee, U.; Weiss, R. G. J. Am. Chem. Soc. 2007, 129, 5012. (e) Khenkin, A. M.; Neumann, R. J. Am. Chem. Soc. 2004, 126, 6356. (f) Khenkin, A. M.; Neumann, R. J. Am. Chem. Soc. 2002, 124, 4198. (g) Li, H.; Li, Z.; Shi, Z. Tetrahedron 2009, 65, 1856. (h) Eisch, J. J.; Dutta, S.; Gitua, J. N. Organometallics 2005, 24, 6291 .

12. (a) Asakawa, Y.; Kondo, K.; Tori, M.; Hashimoto, T.; Ogawa, S. Phytochemistry 1991, 30, 219. (b) Cullmann, F.; Becker, H. Z. Naturforsch., C: Biosci. 1999, 54, 147.
13. Kraut, L.; Mues, R.; Zinsmeister, H. D. Phytochemistry 1997, 45,1249

14. Harinantenaina, L.; Takahara, Y.; Nishizawa, T.; Kohchi, C.; Soma, G.-I.; Asakawa, Y. Chem. Pharm. Bull. 2006, 54, 1046.

15. Apisantiyakom, S.; Kittakoop, P.; Manyum, T.; Kirtikara, K.; Bremner, J. B.; Thebtaranonth, Y. Chem. Biodivers. 2004, 1, 1694.

16. (a) Lee, Y. R.; Choi, J. H.; Yoon, S. H. Tetrahedron Lett. 2005 , 46, 7539. (b) Lee, Y. R.; Lee, W. K.; Noh, S. K.; Lyoo, W. S. Synthesis 2006, 853. (c) Lee, Y. R.; Kim D. H. Synthesis 2006, 603. (d) Lee, Y. R.; Kim, J. H. Synlett 2007, 2232. (e) Wang, X.; Lee, Y. R. Tetrahedron Lett. 2007, 48, 6275. (f) Wang, X.; Lee, Y. R. Synthesis 2007, 3044. (g) Lee, Y. R.; Xia, L. Synthesis 2007, 3240. (h) Lee, Y. R.; Kim, Y. M. Helv. Chim. Acta 2007, 90, 2401. (i) Lee, Y. R.; Li, X.; Kim, J. H.; J. Org. Chem. 2008, 73, 4313. (j) Xia, L.; Lee, Y. R. Synlett 2008, 1643. (k) Lee, Y. R.; Hung, T. V. Tetrahedron 2008, 64, 7338. (1) Lee, Y. R.; Xue, W. Tetrahedron 2009, 65, 10125. (m) Lee, Y. R.; Kim, Y. M.; Kim, S. H. Tetrahedron 2009, 65, 101. (n) Jung, D, H.; Lee, Y. R.; Kim, S. H. Helv. Chim. Acta 2010, 93, 635.

17. Crombie, L. W.; Crombie, W. M. L.; Firth, D. F. J. Chem. Soc., Perkin Trans. 1 1988, 1263.

18. (a) Kurdyumov, A. V.; Lin, N.; Hsung, R. P.; Gullickson, G. C.; Cole, K. P.; Sydorenko, N.; Swidorski, J. J. Org. Lett. 2006, 8, 191. (b) Hubert, C.; Moreau, J.; Batany, J.; Duboc, A.; Hurvois, J.-P.; Renaud, J.-L. Adv. Synth. Catal. 2008, 350, 40. (c) Moreau, J.; Hubert, C.; Batany, J.; Toupet, L.; Roisnel, T.; Hurvois, J.-P.; Renaud, J.-L. J. Org. Chem. 2009, 74, 8963.

19. Lee, H. J.; Lee, Y. R.; Kim, S. H. Helv. Chim. Acta 2009, 92, 1404.

20. Pacher, T.; Segar, C.; Engelmeier, D.; Vajrodaya, S.; Hofer, O.; Greger, H. J. Nat. Prod. 2002, 65, 820.

21. Yang, X.-Z.; Tang, C.-P.; Ke, C.-Q.; Ye, Y. J. Asian Nat. Prod. Res. 2007, 9, 261.

22. Pharmacopoeia of the Peoples Republic of China, English Edition; Chemical Industry Press: Beijing, 2000; Vol. 1, p 199.

23. (a) Do, T. L. Vietnamese Traditional Medicinal Plants and Drugs, 3rd ed.; Publishing House of Medicine: Hanoi, 2001; p 180. (b) Vo. V. C. A Dictionary of Traditional Vietnamese Medicinal Plants; Bio-Pharmaceutical Publisher: Hanoi, 2001; p 162.

24. Kostecki, K.; Engelmeier, D.; Pacher, T.; Hofer, O.; Vajrodaya, S.; Greger, H. Phytochemistry 2004, 65, 99.

25. Yang, X.-Z.; Tang, C.-P.; Ye, Y. J. Asian Nat. Prod. Res. 2006, 8, 4.

26. Adams, M.; Pacher, T.; Greger, H.; Bauer, R. J. Nat. Prod. 2005, 68,83 . 